\title{
Integration of B2B system that supports the management of construction processes with ERP systems
}

\author{
Monika Łobaziewicz \\ OPTeam SA, Tajęcina 113, \\ 36-002 Jasionka \\ Email: mlobaziewicz@opteam.pl
}

\begin{abstract}
B2B is not only a model of cooperation between enterprises. It may also be implemented in companies with a dispersed organizational structure to which construction companies belong. It turns out that B2B systems contribute to an effective implementation of the investments located even in distant geographical locations. The use of Internet technology contributes greatly to the successful exchange of information between the ERP system and other IT systems or applications. Thus, the management of complex processes is facilitated by automating the flow of information and data integration. In construction companies, each investment may be treated as a large and complex project. Therefore, the ERP systems are very useful there. Combined with Web Services with advanced B2B supporting construction processes, they are a powerful tool for managing a construction business.

The purpose of this article is to present the results of an analysis of the idea to integrate ERP systems with OPTIbud system based on research conducted under the second stage of the project "The prototype of an innovative and technologically advanced OPTIbud B2B platform that supports the management of construction processes through the integration of data and information from multiple sources."
\end{abstract}

\section{INTRODUCTION}

$\mathrm{I}$ T TOOLS are used in construction companies all the time, before the beginning of the investment process, during its implementation, and after its completion. In the construction industry, the IT solutions support not only the management of business, financial, and human resources processes, but also the processes typical for the industry, such as tendering, cost estimating, designing, monitoring, standardization, project management, construction works, etc. In the construction industry, the most frequently used systems or applications facilitate design, such as Computer Aided Design (CAD) that helps the architects and designers to quickly and efficiently create a project that requires to make advanced calculations, taking into account investor requirements, different quality standards presented in technical and engineering norms, wide range of building materials and works.

From the effective construction projects management point of view, the system that support the investment process is equally important, in terms of the management and the flow of materials, goods, finances, and human resources. For this purpose, ERP systems integrated with sophisticated IT applications are often used.
Then, the key for the enterprise is whether the data on the progress of implementation of the investment are available in real time. This is important from the decision making point of view. In ERP systems there are mechanisms capable of simulating potential actions and analysing their effects, including analysing financial outcome. This allows, among other things, forecasting, planning, testing, and comparison of possible decision-making activities [1].

ERP systems have a beneficial effect on the functioning of companies. They allow to collect and process data, to make analyses necessary for the development of the whole company as well as for a specific construction project. The author's experience suggests that such a solution proves to be efficient in practice in any company with a complex organizational structure and, in particular, with dispersed structures or organizations that use the model of a remote work, characteristic for construction companies.

ERP systems with dedicated modules or trade applications operating on the basis of a central database connected by mechanisms integrating with other systems, simplify the communication between the construction company and its subcontractors and other business partners or between the headquarter of a construction company and the building site, contribute to cost reduction and the integration of construction processes and the improvement of the quality of services rendered to investors.

For the construction industry, the key aspect is the best combination of the company activities with the implementation of investment projects, often in geographically remote locations, with an intensive rotation and exchange of employees working on construction sites. The right project implementation also requires coordination with many subcontractors and the use of building equipment. Therefore, it turns out that the specific applications or systems supporting construction processes should be integrated with ERP systems. One of the possibilities is B2B system making the use of data stored in ERP system database, processes them, and exports the information back to the base of the system.

In the following section of this article, first the research methodology, then the most popular ERP systems together with the results of the market research are presented. The next sections present the outcome of an integration of ERP systems with other IT systems and applications, methods, architectures, and tools related to ERP systems. 
Each section has some relevant literature and includes conclusions. Finally, the most important recommendations as a result of the study are presented.

\section{RESEARCH METHODOLOGY}

The purpose of this article is to present the results of an analysis of the idea to connect B2B OPTIbud with ERP systems based on research conducted under the second stage of the project entitled "The prototype of an innovative and technologically advanced OPTIbud B2B platform that supports the management of construction processes through the integration of data and information from multiple sources." $[2]^{1}$

To complete this purpose, the following methodology has been adopted. As part of the first stage of the project, the existing computer systems that are being used in the construction industry have been analysed together with indicating the directions of their development in terms of managing the construction processes and technology in which they were created. Then, at this stage, there was conducted in-depth research that covered 10 construction companies. The tool used in the research was a questionnaire consisting of 60 questions regarding the detail lements of a construction process, such as: budgeting, construction manager panel, equipment and transport base, tenders and quoting.

As a result of the research, the designed B2B OPTIbud system should handle the following internal and external processes:

- $\quad$ organization of tenders, including their recording and documents flow,

- budgeting of projects in relation to the most popular programs for cost estimation, such as: Winbud, Rodos, Zuzia, Norma,

- scheduling of construction works

- $\quad$ supporting of the work of a construction manager with the application of data exchange with the ERP system in on-line way,

- settlement of work time of equipment, people as well as fuel usage and building materials,

- handling of the equipment and transport base.

The outcome of the research led to assumptions that are now the basis for the development of the functional architecture of the designed B2B OPTIbud system.

Comparative analysis between the functional expectations of construction companies regarding a dedicated system and the functionalities of computer systems available on the market allowed developing a list of functionality gaps that should be complimented and implemented in the B2B OPTIbud system so that it would create the competitive advantage compared to other solutions.

Based on the results of the first part, the second stage involved an analysis of ERP systems that are most

\footnotetext{
${ }^{1}$ The project, implemented by OPTeam SA is financed from INNOTECH program, HI-TECH path, carried out by The National Centre for Research and Development. The author is the chief scientific officer of the project.
}

frequently used by enterprises from the construction industry, paying special attention to their architecture, functionalities, mechanisms, tools, and capabilities, bearing in mind the aforementioned possibility to integrate the computer solutions with the developed B2B OPTIbud system.

\section{ERP SYSTEMS}

\section{A. The market of ERP systems in Poland and in the world}

According to Gartner, on the global market of ERP systems the leaders are [3]:SAP, Oracle, Sage ERP X3, Microsoft Dynamics AX, Microsoft Dynamics NAV, Infor ERP. In Poland, the market leaders of ERP systems are SAP, Oracle, Comarch, IFS, and BPSC.

According to IDC, in 2012 more than $40 \%$ of market share belonged to SAP [4]. The Comarch was at the second place $(12.5 \%)$, followed by Oracle with $11.7 \%$ of the share. The next two places were occupied by IFS (4.8\%) and BPSC (4.0\%). Analysis of data from previous periods shows that the percentage share of the aforementioned companies has oscillated around these values for several years. Similarly, the position of leading suppliers of ERP systems on the Polish market has not been changed for several years.

Apart from the above mentioned leaders, on the domestic market, there are ERP products of such companies as Asseco Business Solutions, Unit4 Poland, and SIMPLE.

\section{B. The types of implemented ERP systems and methods of their use}

Research conducted by Panorama Consulting Solutions [5] showed that the vast majority of enterprises in 2014 used traditional ERP systems (85\%), and the remaining (15\%) used ERP in a cloud or in SaaS formula. In comparison to 2013, there was a significant decrease in choosing cloud ERP software. In 2013, 26\% of companies have chosen ERP software in the cloud or SaaS compared to $15 \%$ in 2014. Two most important reasons why companies did not implement cloud systems is a lack of knowledge about the technology $(45 \%)$ and the risk and fear of breaches of data security (30\%). The research also shows that the ERP cloud providers usually offer secure and reliable solutions that should be very important for managers in the process of choosing the software.

The research shows that smaller companies want ERP systems to used them in the most important areas of activity, while medium-sized companies expect integration of software with specialized applications in an integrated structure [6].

The important role in ERP systems is played by platforms and technologies for conducting electronic business, and mechanisms that optimize processes within the logistics supply chain [7]. One of the directions of the development of modern ERP systems is taking into account solutions important from the point of view of systems reserved for industries that were previously handled by independent, specialized software. This is primarily about construction 
companies, banks, insurance companies, or companies related to food processing on a small scale [8].

The designers of IT systems are facing new challenges involving the creation and implementation of solutions that support the integration of business processes, including processes specific to a given branch or industry, which would serve as an extension to the functionality of standard ERP management systems [9], [10]. For such solutions to be effective in practice, there must be a suitable mechanism designed for an access to multi-faceted data and information.

\section{INTEGRATION OF SYSTEMS AND APPLICATIONS WITH ERP SYSTEMS}

\section{A. System integration}

Practice shows that the right combination of IT systems in an enterprise is, in many cases, necessary [11].

The system integration has helped to create standards that currently are tools that facilitate the programming works in this scope. High-level programming languages allow to launch created processes with their use programs without modifying various hardware and system platforms. The key role, thanks to its "portability," is played by XML, which is applicable in the domain of data structure description.

Correctly implemented system integration automatizes and improves the processes of the introduction and transfer of data. It is based on making it possible to enable an efficient cooperation of two completely different systems based on different technologies. The answer to these requirements is Electronic Data Interchange (EDI) and B2B solutions.

\section{B. Integration of applications}

In turn, the integration of applications may be analysed in two ways. The first is based on an interaction between applications on the level of understanding the transmitted data bits. Over time, standards were developed which specified higher levels of communication model known as Open System Interconnection Reference Model (OSI RM). These included, respectively, the data-link layer (Ethernet) network (IP), transport (TCP, UDP), and higher layers. This enabled companies that created IT applications to gradually become independent of the elements of communication between the systems, so that they could focus on the semantics of data exchange between integrated elements. At the same time, standards for describing aspects of cooperation between applications were developed. An example of efforts to integrate applications and data within an enterprise that make the sharing of data possible between many heterogeneous computer systems and the integration of a distributed within the enterprise business processes into one coherent set is Enterprise Application Integration (EAI) [12]. On its basis, the Service Oriented Architecture (SOA) pattern was defined that describes integrated systems as independent services cooperating with each other [13],[14].

The attempts to use architectures based on SOA led to the creation of, among other things, the Distributed
Component Object Model (DCOM) and Common Object Request Broker Architecture (CORBA). These technologies had a widespread applicability. However, the implementation of them with the use of SOA with technology proved to be impractical because of the dependence on a particular platform (DCOM) and high complexity (CORBA).

\section{SOA model as an integration tool}

The dynamic development of the Internet has led to the need for integration both between applications inside organizations and between different companies by means of widely used communication protocols. In the construction industry, both types of integration exist. The author's experience leads to the conclusion that the diversity of computer system implementation environments and the pursuit of the reduction of costs associated with the implementation of solutions make the integration an expensive process.

SOA is a widely accepted programming, publishing, searching, and services launching standard. Its basic components include the following [15]:

- Service Provider - responsible for installing the service on the server, publishing its description in the registry and access control;

- $\quad$ Service requester - an application used by the user that discovers services in the registry and orders a demand for their start-up; and,

- Service Registry / Service Broker makes the provision of services possible.

SOA is the architecture for business applications created as a set of stand-alone components, arranged so as to provide services, operating according to certain criteria, and supporting the implementation of business processes [16]. From the end user's perspective, it is a set of services that support the implementation of business processes, and from the technological point of view, it is an infrastructure necessary to provide services.

SOA categorizes relationships between service providers and their customers represented by software components that implement complex business processes. It provides the reuse of software components, the encapsulation of functionality, the precise definition of interfaces, and the flexibility of applications created in a form of composing.

Commonly used element in the implementation of business services based on SOA are Web Services, which are understood as applications that make the business logic available outside the closed area of the network of organizations and which communicate through interfaces used in the Internet and communications protocols.

Web Services [17]:

- are autonomous applications available on the network through their URL (web address),

- have described interfaces and the way of using them by means of structures expressed in XML (Extensible Markup Language),

- are launched by other applications, 
- perform heterogeneous operations ranging from simple responses to user requests to complex business processes,

- use standard communication protocols used on the Internet, and

- provide the possibility to launch and connect with other services providing new functionalities.

Web Services architecture refers to SOA. Therefore, it is based on the following principles:

- Message-oriented;

- Modularity of protocols - the use of the blocks that make up infrastructure protocols, which can then be used in almost any combination;

- The autonomy of services - allowing for independent construction, development, management, progress of versions, and securing endpoints;

- Transparency - control which aspects of endpoint are (or are not) seen by external services;

- Integration based on protocols.

To sum up, the process of integration is based on the interaction of individual systems and applications on the physical or functional level. SOA introduces a model of building computer systems based on communication between separated services. By creating dispersed applications, the individual functions of the program are distinct and separated into separate modules. Subsequently, these segments are merged to form a coherent and dispersed application.

Internet services based on Web Services enabled the effective implementation of the SOA concept in practice. Properly defined and simultaneously unambiguous specification of Web Services that includes, among other things, a description of the communication protocol SOAP and the language of interface description WSDL based on XML, mechanisms of semantic cataloguing and searching for providers caused that this standard quickly gained popularity and is now widely used in dispersed applications. At the same time, thanks to the support of Web Services by coding, language application services have been made available that are inconsistent with SOA through the establishment of appropriate adapters.

Web Services have caused people to move away from the paradigm of the classic integration of applications to the benefit of existing services in processes and their modelling, depending on changing needs.

\section{V.METHODS, ARCHITECTURE AND INTEGRATION TOOLS OF ERP SYSTEMS}

\section{A. SAP and MS Dynamics}

In the case of SAP, B2B system integration may be realized through the following: SAP .NET Connector, Microsoft BizTalk and Duet Enterprise.

The most beneficial tool is SAP.NET, because it may communicate with SAP system in two ways as:
- $\quad$ Client which queries SAP system (server) client calls functions of SAP system and as a reply it receives results,

- $\quad$ Client playing the role of a server, receives calls from SAP while NET program adopts the role of RFC. (The SAP system treats the .NET application code as if it handled a different SAP system. Then, SAP sends a function call to .NET applications and receives results.)

Microsoft Dynamics operates in two versions, as Microsoft Dynamics AX and Microsoft Dynamics NAV. Regardless of which version we are dealing with, the system is delivered to the purchaser, together with its full source code and a set of tools for its development fully integrated with Visual Studio. Integrated Development Environment in MS Dynamics is MorphX that helps to create new modules, windows forms, reports, menus, queries, etc. The application is fully adapted to work with .NET platform. MS Dynamics allows for flexible development and software configuration according to the needs of an enterprise. This gives unlimited possibilities for adapting the system to any organization as well as integration with B2B systems.

The main advantages of the integration of MS Dynamics with other environments are the following:

- Using other technologies to develop the whole enterprise system is possible. Instead of code written in an internal $\mathrm{X}++$ language, it is possible to program functionalities in $\mathrm{C} \#$ and have integration of Microsoft .NET with Microsoft Dynamics;

- MS Dynamics can be coordinated with external applications;

- There is the interaction functionalities and data exchange.

There are a few possibilities to integrate Microsoft Dynamics with other systems:

- The services and application integration platform Application Integration Framework (AIF) and its services make business logic available to external business systems. MS Dynamics platform supports the integration of the AIF platform, using a services programming model. It exposes functionalities by means of services based on Windows Communication Foundation (WCF) from which code may use both MS Dynamics and external programs. WCF services in conjunction with the AIF provide a programming model, tools, and a complete infrastructure to integrate and exchange data using XML.

- .NET Business Connector allows external applications to access Microsoft Dynamics data and using its business logic.

- .NET Interop. allows to cooperate with .NET platform in both directions. It allows $\mathrm{X}++$ code to use the methods managed by the runtime environment of the .NET platform (Common Language Runtime) (CRL). It also provides solutions of proxy classes (C \#, Visual Basic) for Class X ++ MS Dynamics generated in Visual Studio environment, from which C \# code may directly benefit.

Business logic of MS Dynamics is made available through websites that use the latest Windows 
Communication Foundation (WCF) rendered by the Application Object Server (AOS).

The configured pre-processor of requests (Request), AIF, captures all requests. MS Dynamics service runs the necessary business logic to process a request. A similarly configured postprocessor of response (Response) takes over the message of the post-processing response. Then it returns the response to the user.

The best way to integrate the ERP system with external applications is to use services that provide the business logic written in an internal $\mathrm{X}++$ language. In the Microsoft Dynamics environment, the programmer may build, modify, and publish services. Creating a service is based on defining, according to certain rules of $\mathrm{X}++$ class and website interface, and then their linking [18].

Making the services available through the Internet is made through Internet Information Services (IIS), which are equivalent to Web Services, and which redirect requests to the AOS regardless of whether they come from the Internet or intranet. AOS returns responses for the recipient of the service through IIS. The exchange configured to the use of Web Services is not queued, since it is supported synchronously.

AIF provides an extensible platform for the exchange of documents with external systems using XML technology. Both the synchronous and asynchronous transmission is possible. In the case of the synchronous exchange, the requests are paired with the answers, which means that the same connection it is used and that the demanding party does not continue work but just waits for a response. AIF immediately processes requests and sends responses. In asynchronous mode, the requests are queued, and the answers are sent with a time delay. The advantage is a more efficient processing of a large number of messages.

AIF supports the exchange in both directions: incoming and outgoing. Incoming exchange means that the external system, for example, B2B may send commands. On the other hand, outgoing is receiving data coming from the MS Dynamics system and sending them to the requesting user.

\section{B. $C D N X L$}

The Comarch ERP XL system allows an interactive work with the user and also acts as an application server that provides external applications' built-in functionalities. This is provided by using a function mechanism, called the Application Programming Interface (API).

The external application may connect to $\mathrm{CDN}$ XL application server by means of API and use mechanisms available to the ERP system. This provides the openness of the system, making the integration possible with standard Microsoft Visual Studio programming tools and any external system. The CDN XL system provides an access to a dozen of API functions stored in the CDN_API.dll library supplied with the system.

The XL-API mechanism is used by IT companies who form their own solutions that cooperate with the Comarch ERP XL system. It allows for simplicity and security through the use of direct record in database and independence from the system and database platform, on which Comarch ERP XL runs.

The CDN XL system, while implementing the security policy, logs the changes to the database via the API functions. If an error occurs causing the termination of the application that calls out API functioning, there is a reenactment of a database to the state before running the API function.

Approval of the transaction occurs upon the termination of entering the object or log- ut function (XL Logout).

XL API functionality with the development of CDN XL system is constantly being expanded. In order to ensure compatibility with previous API versions, there was introduced a versioning mechanism. It serves as a translator between API calls from external applications and structures used inside CDN XL system. For each version of the system, there is a corresponding version of the API libraries that allows the integration with it.

The programmer using the API functions defines its version based on which its structure is recognized and the transfer of the value to the internal CDN XL structure is made. In the case of non-compliance of structures, unused fields are filled with default values. The versioning mechanism is bi-directional. Data structures used by API also allow the return of data from CDN XL using the translation procedures.

\section{RECOMMENDATIONS CONCERNING INTEGRATION}

Based on the research of ERP systems and standards, integration tools, the following actions are recommended for the B2B OPTIbud platform:

- Implementation of mechanisms enabling a direct connection to the database of the ERP system,

- The use of Web Services available for ERP systems,

- Direct use of different ERP systems with a business logic characteristic in construction processes,

- Using an interface that is made available by the ERP system,

- The transfer of files with data using XML formats.

Moreover, there were identified the following activities characterising the impact of integration complexity requirements and architecture (Fig. 1).

The scale of the flow of various data and information is so large that it is difficult for companies to function on autonomous systems or computer applications. Integration complexity positively influences the optimization of business processes, enables a seamless cooperation of organizational units, and gives a possibility to operate on a one database to all participants of the processes work on the same actual data.

In many construction companies, heterogeneous applications coexist. The technological progress and market demands caused that construction companies carry out their operational activities through the Internet, and that is why the ERP software must cooperate with many applications. Considering the above, one of the key conditions for the 


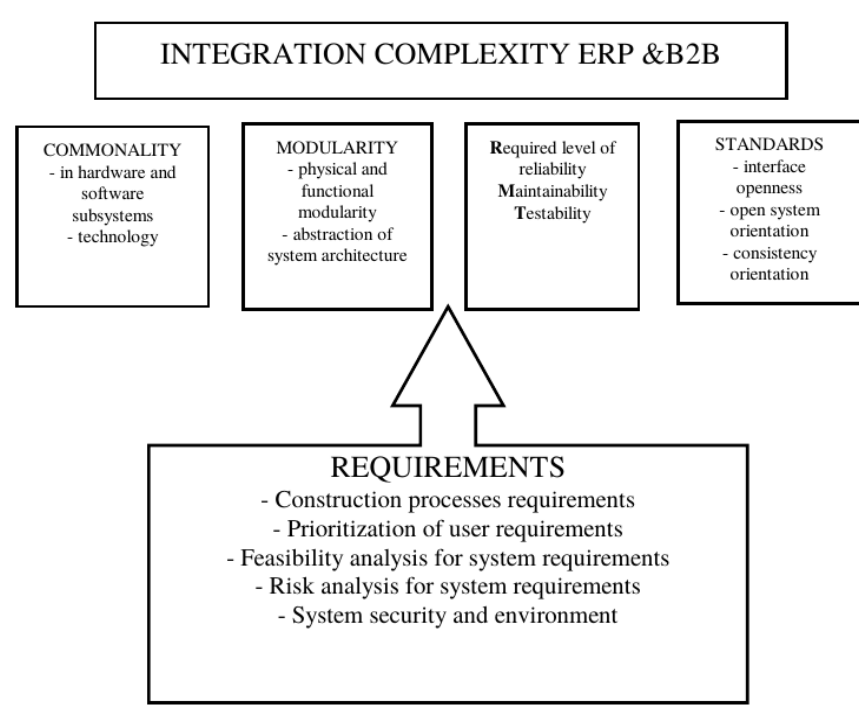

Fig. 1. Attributes having the impact on ERP system with B2B OPTIbud integration complexity

effectiveness of B2B OPTIbud is its integration with ERP systems end points. In this case, the interaction in the scope of data, information, and construction processes takes place on many levels.

The OPTIbud B2B system will be based on the philosophy of integrated systems that guarantees the flow of information between the individual modules in the ERP system, so the user would not have to repeatedly enter the same data in different functional areas of the system, but he will use the data already entered, transforming them into information necessary to make decisions and execution of construction processes. Thus, the B2B OPTIbud system, because of its autonomy may be able to be integrated with various ERP systems operating on the market. Thanks to built-in mechanisms, it would be able to communicate automatically with the database of the ERP computer system of a construction company.

\section{REFERENCES}

[1] W.M. Grudzewski, I. K. Hejduk, Methods of management systems design. Warszawa: Difin, 2004.
[2] OPTeam SA, The prototype of an innovative and technologically advanced OPTIbud B2B platform that supports the management of construction processes through the integration of data and information from multiple sources, Project documentation.

[3] The market of ERP class systems ERP. http://issuu.com/benchmarkpl/docs/bbm_nr5, p.21 (Retrived 10.04.2015)

[4] http://biznes.benchmark.pl/artykul/rynek-systemow-klasy-erpraport/strona/230 (Retrived 30.03.2015)

[5] Panorama Consulting Solutions ,2014 ERP Report”, http://panoramaconsulting.com/resource-center/2014-erp-report/ (Retrived 8.04.2015)

[6] http://www.erp-view.pl/erp/koniec_erp.html (Retrived 5.04.2015)

[7] B. Wieder, P. Booth, et. al, "The impact of ERP systems on firm and business process performance", Journal of Enterprise Information Management, Vol. 19 Iss: 1, 2006, pp.13-29, http://dx.doi.org/10.1108/17410390610636850.

[8] A. Chandrasekaran, G.Elias, R.Cloutier, R.Jain. 'Exploring the Impact of Systems Architecture and Systems Requirements on Systems Integration Complexity", IEEE Systems Journal, vol. 2, no. 2, pp. 209223, June 2008

[9] J. Ram, D. Corkindale, M.L. Wu, ,Implementation critical success factors (CSFs) for ERP: Do they contribute to implementation success and post-implementation performance?", International Journal of Production Economics, Volume 144, Issue 1, July 2013, pp. 157-174, doi:10.1016/j.ijpe.2013.01.032.

[10] J. May, G. Dhillon, M. Caldeira, "Defining value-based objectives for ERP systems planning”, Decision Support Systems, Volume 55, Issue 1, April 2013, pp. 98-109, doi:10.1016/j.dss.2012.12.036.

[11] S. Durvasula, M. Guttmann, A. Kumar, J. Lamb, T. Mitchell, B. Oral, Y. Pai, T. Sedlack, H. Dr Sharma, S. Ram Sundaresan, "SOA Practitioners' Guide. Part 1: Why Services-Oriented Architecture?", Oracle, 2006.

[12] R.E Giachetti, "A Framework to review the Information Integration of the Enterprise", International Journal of Production Research, vol. 42,no.6,2004, pp.1147-1166, doi: 10.1080/00207540310001622430.

[13] R. Jardim-Goncalvesa, A. Grilob, A. Steiger-Garcaoa, "Challenging the interoperability between computers in industry with MDA and SOA", Computers in Industry, Volume 57, Issues 8-9, December 2006, pp. 679-689, doi:10.1016/j.compind.2006.04.013.

[14] T. R. Soomro, A. H. Awan, "Challenges and Future of Enterprise Application Integration", International Journal of Computer Applications (0975 - 8887), vol. 42, no.7, March 2012.

[15] S. Gandhi, "A Service-Oriented Approach to B2B Integration Using Web Services", White Paper Published for Dreamscape Media, http://www.featbooks.com/read-online/a-service-oriented-approach-tob2b-integration-using-web (Retrived 20.03.2015)

[16] J. Łagowski, SOA - ideology, not technology, XV Conference PLOUG, Kościelisko, 2009, pp.180-192.

[17] M. Pielecka, ,Integration of computer systems - interorganisational information exchange ", Journal of Management and Finance, vol. 28, no. 4, part 1., 2013.

[18] http://msdn.microsoft.com/en-us/library/aa877498.aspx (Retrived 10.04.2015). 